\title{
Physicochemical Properties of Causative Drugs Associated with Renal Nephrotoxicity
}

\author{
Reiko Yano ${ }^{1^{*}}$, Fumiko Ohtsu ${ }^{2}$ and Nobuyuki Goto ${ }^{3}$ \\ ${ }^{1}$ College of Pharmacy, Kinjo Gakuin University, Japan \\ 2Pharmaceutical Information Center, Faculty of Pharmacy, Meijo University, Japan \\ ${ }^{3}$ Drug Informatics, Faculty of Pharmacy, Meijo University, Japan
}

\begin{abstract}
Background: In this study, we examined the association between drug-induced renal nephrotoxicity and the physiochemical properties of the causative drugs we extracted from CARPIS in order to provide drug information about potential severe risks at the time of and after marketing approval.

Methods: We designated the nephrotoxicity-associated drugs as the case drug group (126 drugs), and all other drugs were set as the control drug group (915 drugs). We compared the physicochemical properties of the group. We performed univariate logistic regression analysis on each investigation item, and then performed multivariate stepwise logistic regression analysis on the items which were $p<0.2$ using univariate logistic regression analysis.

Results: Model 1, which uses a logP value, showed that the odds ratio of pKa at less than 7 was 2.46 , and that at less than 7-8 was 2.01. The odds ratio of logP value at less than 0 was 1.67 . MW at less than $300-400$ was 0.57 . Model 2, which uses a $\log \mathrm{D}$ value, showed that $\log \mathrm{D}$ at less than 0 was 2.23 . The odds ratio of pKa at less than 7 was 2.34 , and at less than 7-8 was 2.04. The odds ratio of MW at less than $300-400$ was 0.56 .

Conclusion: The results clearly showed the risk of renal problems associated with water-soluble drugs. The information could be useful to consider potential risks of renal problems associated with water-soluble drugs at the time of drug approval and after marketing and to compensate for the lack of information.
\end{abstract}

Keywords: Adverse drug reactions; Drug-induced renal damage (nephrotoxicity); Physicochemical property; Lipophilicity; LogD

Abbreviations: ADR: Adverse Drug Reactions; MW: Molecular Weight; HDO: Hydrogen Donors; HAC: Hydrogen Acceptors

\section{Introduction}

Drugs can have both unfavorable adverse drug reactions (ADR) and useful pharmacological effects. Previous studies have reported various ADR and toxic effects caused by drugs [1]. Those reports noted that some products were banned after being put on the market because of the severe ADR they caused [2], while the safety of others is assured by having usage restrictions imposed on them [3].

It is important not only to have a response that forms countermeasures when ADR emerge, but also to provide drug information to form safety measures to predict and avoid ADR before they occur.

There are 3 essential pieces of information about specific pharmaceutical products: physiochemical, pharmacological, and pharmacokinetic. Thus, various pharmacological and pharmacokinetic approaches that examine the association between these 3 pieces of information and adverse events caused by drugs have been previously reported [4-6]. However, there are few reports that examine the association between physiochemical information and adverse events.

Drug-induced renal nephrotoxicity can affect the glomerulus, renal tubules, renal vasculature, and ureter, and its mechanisms of development could be generally divided into toxic and allergic mechanisms $[7,8]$. Kidneys are small organs comprising less than $1 \%$ of total body weight, and they, like the liver, are vulnerable to drugs. The reason for that is: 1) $20-25 \%$ of cardiac output flows into the kidneys; thus, a large amount of blood is exposed to drugs, 2) drugs are easily absorbed into the epithelial cells with the aid of transporters, and 3) high concentrations of drugs accumulate in the kidneys because $\mathrm{pH}$ changes in primitive urine increase the effects of non-dissociative drugs and accelerates the drug's migration via passive diffusion [9].

This study examined the association between drug-induced renal nephrotoxicity and the physiochemical properties of the causative drugs in order to provide drug information where it is inadequate as well as providing information about potential severe risks at the time of and after marketing approval.

\section{Materials and Methods}

\section{Subjects of investigation}

We utilized the "Collection of Drug Formulae in Japan, 2011" (Japan Pharmaceutical Information Center) which contains prescription drugs available in Japan and includes 1,340 ingredients. Of those 1,340 ingredients, we examined 1,041 drugs excluding the following drugs: compound drugs (77 drugs); potassium calcium agents (8 drugs); gold or platinum-containing agents (10 drugs); drugs which cannot be calculated from available data on physicochemical properties such as: coordinate bonds (12 drugs), radiopharmaceuticals (9 drugs), polymers ( 28 drugs), drugs for which it is technically impossible to give

*Corresponding author: Reiko Yano, Kinjo Gakuin University, College of Pharmacy, 2-1723 Omori, Moriyamaku, Nagoya, Aichi 463-8521, Japan, Tel: +81 52798 0180; Fax: +81 52798 0754; E-mail: reiya@kinjo-u.ac.jp

Received November 17, 2015; Accepted December 07, 2015; Published December 11, 2015

Citation: Yano R, Ohtsu F, Goto N (2015) Physicochemical Properties of Causative Drugs Associated with Renal Nephrotoxicity. J Pharmacovigil 3: 189 doi:10.4172/2329-6887.1000189

Copyright: ( 2015 Yano R, et al. This is an open-access article distributed under the terms of the Creative Commons Attribution License, which permits unrestricted use, distribution, and reproduction in any medium, provided the original author and source are credited. 
structural formulae (13 drugs); drugs that have the same formulae (17 drugs); diagnostic agents/agents for public health/agents for dispensing use (31 drugs).

\section{Investigation items}

1,041 drugs were divided into Molecular Weight (MW), pKa, Hydrogen Donars (HDO), and Hydrogen Acceptors (HAC). The drugs are categorized as anion, neutral, zwitterion, and cation with the charge status set at pH7.4. The main information sources we drew from are the drug interview form of each drug and ACD/Labs software Ver. 12.5 (FUJITSU Technical Computing Solution). We selected a pKa value that is the closest to physiological pH7.4, without dividing it into acid base or conjugate acid. We designated the charge status as pH7.4 using $\mathrm{ACD} / \mathrm{Labs}$ software and applied the drug formulae to it. We performed our calculations with the $\log \mathrm{P}$ and $\log \mathrm{D}$ values at $\mathrm{pH}$ 7.4. $\log \mathrm{P}$ is a value that shows the non-dissociative partition coefficient as well as the lipophilicity of drugs. On the other hand, $\log \mathrm{D}$ is a value of the non-dissociative partition coefficient. Drugs are disassociated due to $\mathrm{pH}$ changes and this can influence calculations, but this influence can be avoided by using $\log \mathrm{D}$. Drug interview forms in Japan usually apply $\log \mathrm{D}$ value. However, the $\log \mathrm{P}$ value is necessary to analyze the association between the levels of lipid solubility in drugs and druginduced renal nephrotoxicity. Therefore, we applied both $\log \mathrm{P}$ and $\log \mathrm{D}$ values to the partition coefficients in this study.

We analyzed the distribution data and divided it into 25/50/75 percentile quarters: MW (less than 300, less than 300-400, less than 400-500, and more than 500); pKa (less than 7, less than 7-8, less than $8-9$, and more than 9 ); HDO ( 1 or less, $2 / 3 / 4$ or more); HAC ( 4 or less, $5 / 6 / 7$ or more). The charge status is anion, neutral, zwitterion, and cation. We also divided $\log \mathrm{P}$ and $\log \mathrm{D}$ values into 2 categories: less than 0 and 0 or more.

\section{Extraction of the case drug group and comparative investiga- tion}

We used a database called Case Report of Adverse Drug Reaction and Poisoning Information System (CARPIS) to extract cases of druginduced renal nephrotoxicity (case drug group). CARPIS is a database system that collects adverse drug reactions (ADR) and intoxication, which was created at the Pharmaceutical Information Center, Faculty of Pharmacy, Meijo University. The data has been exhaustively collected from case reports about ADR and intoxication that have been published in Japanese academic journals and presented at academic conferences since 1987. CARPIS enables researchers to search case reports for ADR by searching terms such as 'combination drugs' or 'patient background' [10]. Approximately 75,000 references are available to search as of 2015. Of the case reports of drug-induced renal nephrotoxicity registered in CARPIS, 476 case reports which met "Case Report Record Priorities and Evaluation Criteria" and "Causality Relationship of Adverse Drug Events and its Evaluation Criteria" and were evaluated as above certain levels were extracted [10]. Drug-induced renal nephrotoxicity in this study includes the following diseases: acute renal failure, glomerular nephritis, interstitial nephritis, and tubular disorder. On the other hand, the following diseases were excluded from consideration in this study: postrenal renal failure such as urinary lithiasis; secondary renal nephrotoxicity including rhabdomyolysis and malignant syndrome; and chronic renal failure. 126 causative drugs in case reports of druginduced renal nephrotoxicity were extracted and assigned to the case drug group. 915 drugs out of 1,041 drugs of interest (except the drugs in the case drug group) were assigned to the control group.

\section{Statistical analysis}

We compared the mean values of the case drug group and the control drug group, and set the reference as minimum value when the mean value in the case drug group is large and maximum value when the mean value in the control group is small. We performed univariate logistic regression analysis for each item of both the case drug group and the control drug group. To investigate the factors that could be associated with drug-induced renal nephrotoxicity, we set the items that were $\mathrm{p}<0.2$ as an independent variable and performed logistic regression analysis to calculate $\mathrm{p}$ value. Both the case drug group and control drug group were assigned to dependent variables. We performed multivariate stepwise logistic regression analysis on the physicochemical-property data of the drugs. The significance level was $\mathrm{p}=0.05$ when the model took a variable number, and $\mathrm{p}=0.10$ when the variable number was removed.

$\log \mathrm{P}$ values were assigned to Model 1 , and $\log \mathrm{D}$ values were assigned to Model 2 in order to avoid multicollinearity because both $\log \mathrm{P}$ and $\log \mathrm{D}$ values revealed high correlations of $0.875(\mathrm{p}<0.01)$ after being analyzed by Spearman's correlation coefficient.

Charges that were difficult to extract from drug interview forms were excluded from multivariate logistic regression analysis. We used SPSS Statistics 22 software (IBM) for the statistical analysis, and significance level was set at 0.05 (5\%) unless otherwise stated.

\section{Results}

\section{Item characteristics}

The MW in the case drug group (126 drugs) was 92.09-1,473.59, $396.11 \pm 250.83$ (range, mean \pm SD). $\log P$ value was $-5.26-7.64,1.45 \pm$ 2.57; $\log \mathrm{D}$ value was $-10.67-7.64,-0.34 \pm 3.16$; $\mathrm{pKa}$ was $0.52-13.94,6.60$ \pm 3.11 ; HAC was $1-39,8.08 \pm 6.03$; HDO was $0-26,3.57 \pm 4.12$.

On the other hand, the MW in the control drug group (915 drugs) was 42.04-2,933.44, $374.29 \pm 194.06$ (range, mean \pm SD). The $\log P$ value was $-9.21-19.12,2.10 \pm 2.71$; the $\log \mathrm{D}$ value was $-21.96-19.12$, $0.81 \pm 3.30$; $\mathrm{pKa}$ was $0.08-13.92,7.46 \pm 2.98$; HAC was $0-71,6.38 \pm 5.18$; HDO was 0-50, $2.46 \pm 3.24$.

\section{Univariate logistic regression analysis}

We performed univariate logistic regression analysis with the case drug group and the control drug group as dependent variables and $\mathrm{MW}, \mathrm{pKa}, \mathrm{HDO}, \mathrm{HAC}$, charge status, and coefficients $(\log \mathrm{P}$ and $\log \mathrm{D})$ as independent variable, and show the results in Figure 1. The MW, $\log \mathrm{P}, \log \mathrm{D}, \mathrm{pKa}, \mathrm{HAC}, \mathrm{HDO}$, charge were $\mathrm{p}<0.2$.

\section{Multivariate logistic regression analysis}

We performed multivariate logistic regression analysis with items that showed significance $(p<0.2)$ as independent variables, and the case drug group and the control drug group as dependent variables in order to adjust the confounder of each item and drug-induced renal nephrotoxicity. The physicochemical properties of drugs were analyzed by stepwise logistic regression analysis.

$\mathrm{pKa}, \log \mathrm{P}$, and $\mathrm{MW}$ in that order were selected as highly relevant data in Model 1 using the $\log \mathrm{P}$ value (Figure 2), whereas HAC and HDO were excluded. The reference value for pKa was set at above 9 . The odds ratio at less than 9 was 2.46 (95\% CI: 1.51-4.03, p=0.000) and 2.01 (95\% CI: 1.01-4.00. $\mathrm{p}=0.048)$ at less than 7-8. As a result, the association of $\mathrm{pKa}$ with drug-induced renal nephrotoxicity was significantly high. The odds ratio of the $\log \mathrm{P}$ value at less than 0 when 


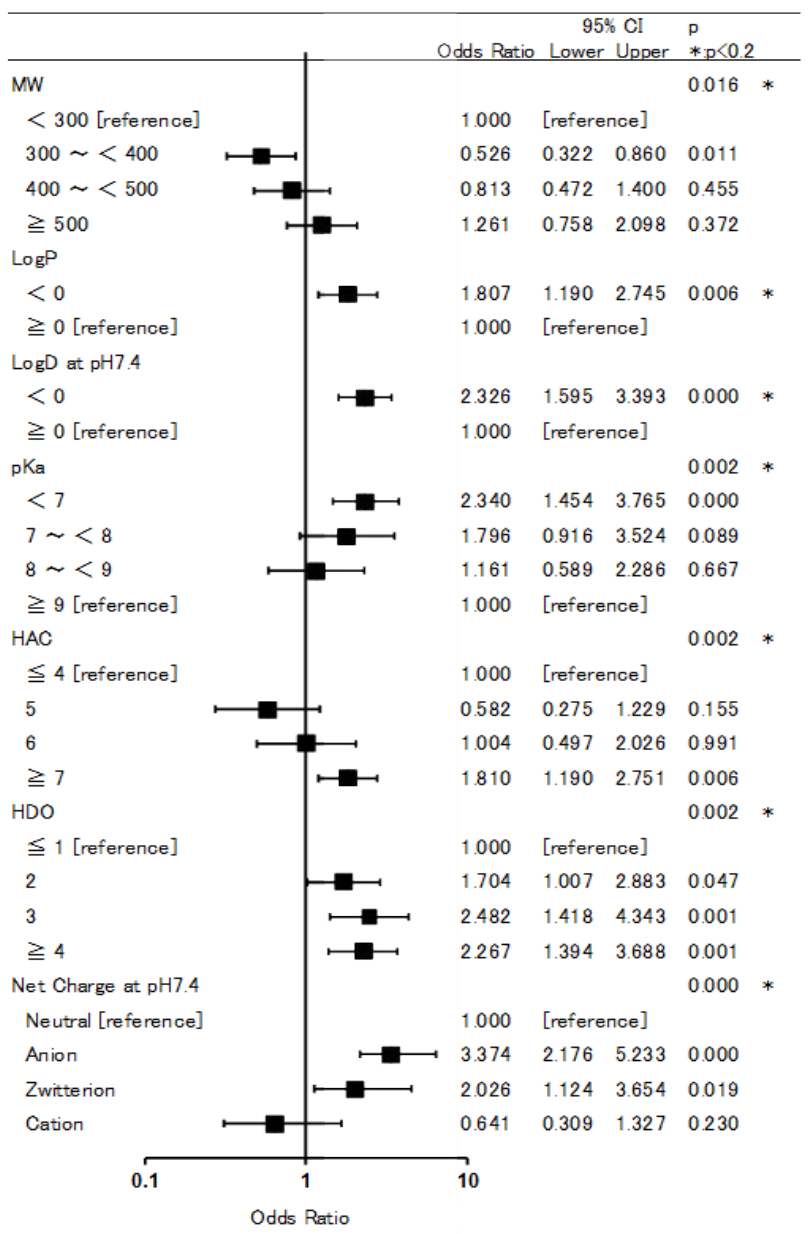

Abbreviation: $\mathrm{MW}=$ molecular weight; $\mathrm{HDO}=$ hydrogen donors; $\mathrm{HAC}=$ hydrogen acceptors

Figure 1: Univariate logistic regression analysis of the physicochemical properties of the causative drugs.

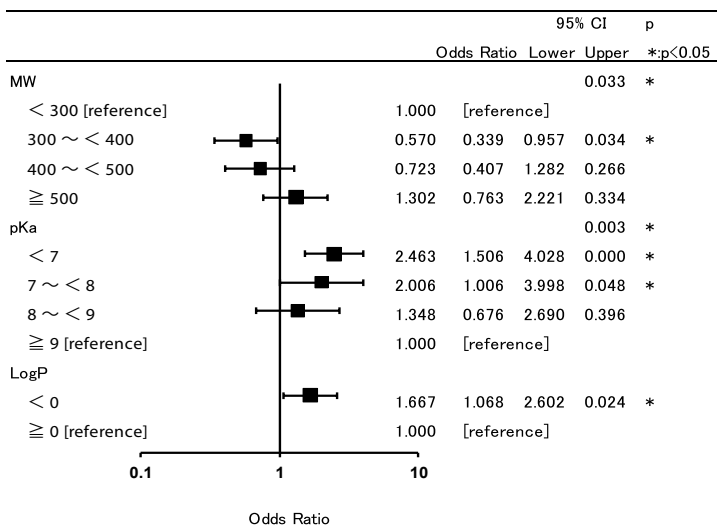

Abbreviation: $\mathrm{MW}=$ molecular weight; $\mathrm{HDO}=$ hydrogen donors; $\mathrm{HAC}=$ hydrogen acceptors

The partition coefficient is $\log \mathrm{P}$.

Figure 2: Multivariate logistic regression analysis of the physicochemical properties of the causative drugs utilizing $\log \mathrm{P}$. comparing with it the $\log \mathrm{P}$ value at more than 0 was 1.67 (95\% CI: $1.07-2.60, \mathrm{p}=0.024)$; thus, it became evident that the association with drug-induced renal nephrotoxicity and $\log \mathrm{P}$ was high. The odds ratio of the MW at less than 300-400 when comparing with the MW at less than 300 was 0.57 (95\% CI: 0.34-0.96, p=0.034); thus, the association of MW with drug-induced renal nephrotoxicity was low.

$\operatorname{LogD}, \mathrm{pKa}$, and $\mathrm{MW}$ in that order were selected as highly relevant data in Model 2 using the $\log \mathrm{D}$ value (Figure 3), whereas HAC and HDO were excluded. The odds ratio of $\log \mathrm{D}$ at less than 0 was 2.23 (95\% CI: 1.49-3.33, $\mathrm{p}=0.000$ ) when compared with the $\log \mathrm{D}$ value at more than 0 ; thus, the association of the items with drug-induced renal nephrotoxicity was significantly high. The odds ratio of $\mathrm{pKa}$ at less than 7 was 3.34 (95\% CI: 1.42-3.84, p=0.001) when compared with $\mathrm{pKa}$ at more than 9; thus the association of $\mathrm{pKa}$ with drug-induced renal nephrotoxicity was significantly high. The odds ratio of the MW at less than $300-400$ was 0.56 (95\% CI: 0.33-0.93, p=0.026); thus, it became evident that the association of the MW value with drug-induced renal nephrotoxicity was low compared to the MW at less than 300 .

\section{Discussion}

The physicochemical property data of each drug is derived from formulae and therefore the data of each drug is related [11]. To elucidate the association between drug-induced renal nephrotoxicity and physicochemical property data, we investigated the physicochemical property data of drugs, performed univariate logistic regression analysis on cases of drug-induced renal nephrotoxicity (the case drug group) and those without it (the control drug group), and performed multivariate analysis on the associations between the groups in order to objectively summarize the results.

Using the $\log \mathrm{P}$ and $\log \mathrm{D}$ values, we found that the odds ratio of water-soluble drugs was approximately 2 and the odds ratio of $\mathrm{pKa}$ at less than 8 was approximately 2 ; thus, it became clear that the association of water-soluble drugs and $\mathrm{pKa}$ value with drug-induced renal nephrotoxicity was high. The odds ratio of MW at less than $300-$ 400 was approximately 0.6 ; thus, the association of MW with druginduced renal nephrotoxicity was low. Renal excretion of drugs is completed in 3 steps: glomerular filtration, secretion in the proximal tubule, and reabsorption in the distal tubule. Substances passing through the glomerular pores sized 70-100 $\AA$ and with a MW up to 5,500 are almost completely filtered. However, albumin, which is negatively charged and has a MW of approximately 68,000 , is less likely to be filtered through the glomerular membrane $[12,13]$. The MW of the drugs we extracted that are associated with renal nephrotoxicity was 92.1 at minimum value (glycerin), 1473.6 at maximum value (peplomycin), and the range of glomerular filtration (70-100 $\AA$ ). On the other hand, in the process of tubular secretion or reabsorption, subject drugs as transporters might be related to molecular weight; for instance, OAT transports highly-hydro soluble substances at a MW of 500 or less [14]. The odds ratio of the MW at 300-400 was low (0.56-0.57) in this study. Because of this, our study suggests an association between membrane permeability and drug-induced renal nephrotoxicity based on intracellular accumulation, although no research has been done on the association between drug-induced renal nephrotoxicity and MW. Further in-vitro study is required.

Generally, because water-soluble drugs are likely to be eliminated through renal excretion, drug concentration in the kidneys is likely to be high according to the examination of the partition coefficients $(\log \mathrm{P}$, $\log \mathrm{D})$. On the other hand, 1) liposoluble drugs are passively diffused from outside of the renal tubules, reducing the efficacy of elimination 2) 


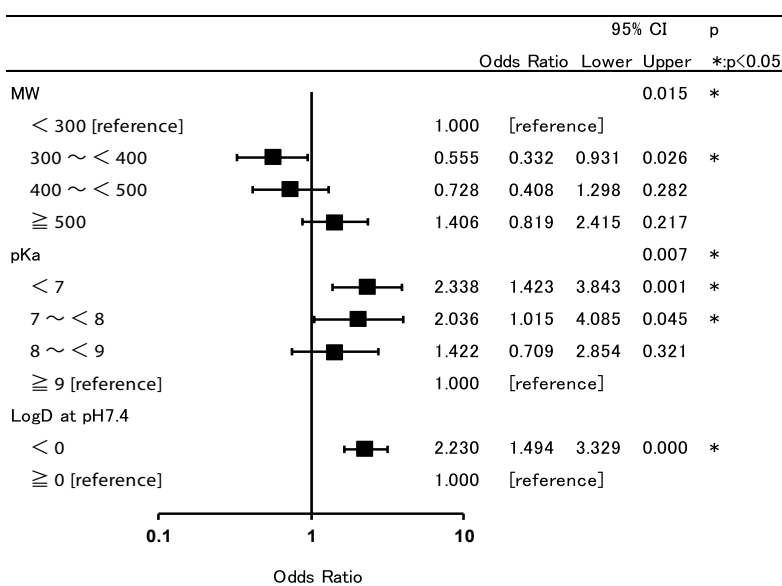

Abbreviation: $\mathrm{MW}=$ molecular weight

The partition coefficient is $\log \mathrm{D}$.

Figure 3: Multivariate logistic regression analysis of the physicochemical properties of the causative drugs utilizing $\log \mathrm{D}$.

while drug concentration in the primitive urine, which is filtered from the glomerulus, has a greater density than perivascular space because of the reabsorption of water. 3) Finally, highly-liposoluble drugs are likely to be metabolized in the liver $[12,13]$.

For example, 46 drugs out of the drugs associated with nephrotoxicity we extracted are antipyretic analgesic antiphlogistics. There are 9 drugs in the case drug group $(\log \mathrm{D}$ at less than 0$)$ and 10 drugs at more than 0 , and 5 drugs at less than 0 in the control drug group and 22 drugs at more than 0 ; therefore, the case drug group shows a higher proportion of water-soluble drugs than the control drug group. The mean values are -0.18 in the case drug group and 1.5 in the control drug group; therefore, the partition coefficient in the case drug group showed a lower mean value.

We predicted that water-soluble drugs, which increase urine concentration, are likely to cause renal dysfunction; however, in this study, the odds ratio obtained by using $\log \mathrm{P}$ was 1.67 , and that obtained by using $\log \mathrm{D}$ was 2.23 , which was almost double. Therefore, we could objectively summarize the data, which showed that water-soluble drugs are likely to cause drug-induced renal nephrotoxicity when compared to fat-soluble drugs. The difference between $\log \mathrm{P}$ and $\log \mathrm{D}$ is that 1) $\log \mathrm{P}$ is a value that shows the partition coefficient of non-dissociative drugs; on the other hand, 2) $\log \mathrm{D}$ is partition coefficient that considers dissociative influence being affected by changes in $\mathrm{pH}$. Currently, the partition coefficient values that are available to obtain from package inserts or drug interview forms are not unified and are varied in measurement methods. However, the descriptions of the partition coefficient in the drug interview forms came to be unified to describe every $\mathrm{pH}$ as 1-octanol/water in 2013. Therefore, hereafter, it is expected that partition coefficients will be described as such, and predictions and comparisons using physicochemical property data that describes every $\mathrm{pH}$, will become easier.

In the process of passive diffusion inside of the body drugs are likely to depend on $\mathrm{pH}$ partition theory. Gastrointestinal absorption of drug was predicted during research and development and tends to be taken into account using the $\log \mathrm{D}$ value that considers $\mathrm{pH}$ within the gastrointestinal tract [15]. We also found that $\log \mathrm{D}$ is considered to be more appropriate for the prediction of drug-induced renal nephrotoxicity because $\log \mathrm{D}$ showed a higher odds ratio than $\log \mathrm{P}$.

It is not easy to classify pKa into acid or conjugate acid. Therefore, we applied a pKa value of nearly 7.4 when multiple pKa was found in drugs. In this study, the odds ratio of drugs with a pKa value of less than 8 was doubled. Our examination of the charge related to $\mathrm{pKa}$, showed that the odds ratio of anionic drugs was 3.37, and that of amphoteric drugs was high at 2.03 .

According to a previous report, renal tubular secretion is affected by the ratio of non-bonding and the transporters and it is an important eliminatory mechanism for almost half of the drugs that show their ion formation by their physiological $\mathrm{pH}$ [16]. Anion transporters and cation transporters are inherent in human kidneys; anionic drugs are mediated by anion transporters, and cationic drugs are mediated by cation transporters, and ampholytic drugs are mediated by anionic transporters in order to be excreted into the urine [17]. In this study, the odds ratios of anionic and ampholytic drugs were high. However, it is necessary for anionic and ampholytic drugs to flow against the potential electric difference in order to be taken from the renal basement membrane into the cell. Hence, our study suggested that there are some associations between the transporters concentrating drugs in the cells and drug-induced renal nephrotoxicity. The association between them was suggested in the univariate logistic regression analysis on $\mathrm{HAC}$ and HDO, but HAC and HDO were not selected in the multiple logistic regression analysis. Because of this the association between transporters concentrating drugs in the cells and drug-induced renal nephrotoxicity was considered low.

We used CARPIS for the case drug group in order to extract drugs that are clearly associated with drug-induced renal nephrotoxicity and therefore did not need to use large-scale databases that included spontaneous ADR reports. Spontaneous ADR reports in large-scale databases are often ambiguous about the reliability of data and the causal associations between data and drugs [18]. On the other hand, many ADR that can be problematic in clinical practice have been reported in CARPIS. Moreover, the causal associations have been investigated clearly, and the information is sufficient. However, there are limitations to CARPIS: 1) there is a time lag, 2) minor ADR are unlikely to be reported, and 3) we cannot investigate every possible adverse reaction reported. Those factors could lead to underestimating the risk of the drugs we extracted that are associated with nephrotoxicity.

It is necessary to develop appropriate strategies to manage this possible risk during the development of a drug and after it goes on the market in order to ensure safety. It is possible that the 7 types of data in this study were not sufficient for complete analysis, but we applied all available and practical data from the package inserts, drug interview forms, and structural formulae. We suggest that the data is considered sufficient to prevent potential risks and compensate for the lack of information at the time of drug approval or after marketing.

\section{References}

1. Eguchi R, Kato M, Kaneko E, Kusaba K, Yoshikawa M, et al. (2015) A systematic review of decided litigated cases on adverse drug events in Japan: classification of decided cases appearing in law reports. Yakugaku Zasshi 135: 501-506.

2. Watabe T, Ogura K, Nishiyama T (2002) Molecular toxicological mechanism of the lethal interactions of the new antiviral drug, sorivudine, with 5 -fluorouracil prodrugs and genetic deficiency of dihydropyrimidine dehydrogenase. Yakugaku Zasshi 122: 527-535.

3. Chugai Pharmaceutical Co., Ltd.: Urgent safety information: Abnormal behavior after taking Tamiflu. https://www.pmda.go.jp/files/000147877.pdf (accessed 1 August 2015) 
Citation: Yano R, Ohtsu F, Goto N (2015) Physicochemical Properties of Causative Drugs Associated with Renal Nephrotoxicity. J Pharmacovigil 3: 189. doi:10.4172/2329-6887.1000189

4. Sawada Y (2000) Quantitative prediction of the pharmacological effects and adverse reactions based on pharmacokinetics and pharmacodynamics. Drug Metabolism and Pharmacokinetics 15: 386-395.

5. Tanaka M, Orii T, Kobayashi K, Hirono S (2001) Examination of factors affecting efficacy and adverse effect, for the retrospective study of vancomycin hydrochloride (VCM). Yakugaku Zasshi 121: 621-629.

6. Nagata M (2015) Clarification of Mechanisms and Risk Factors of Adverse Drug Reactions Using a Pharmacokinetic-Pharmacodynamic Analysis. Japanese Journal of Pharmaceutical Health Care and Sciences 41: 205 -214.

7. Takeuchi Y, Abe T (2013) Suspect drug-induced renal nephrotoxicity from symptoms and pathophysiology. The Pharmaceuticals 55: 2329-2333.

8. Hirata S, Kadowaki D, Narita Y (2012) Drug-induced renal nephrotoxicity and the proper use of pharmaceutical products. The Japanese Journal of Nephrology 54: 999-1005.

9. Ueda S (1998) Drugs that cause renal dysfunction and its mechanism. The Pharmaceuticals 40: 769-785.

10. Ohtsu F, Yano R, Inagaki K (2006) Development of a facts data-base for evidence-based adverse reactions diagnosis. Drug Information Journal 40: 115-122.
11. Kouzuki H (2009) Development of an In Silico Prediction System for the Risk Assessment of Chemicals -Development of a Prediction Model for Skin Irritation. Journal Society Cosmetic Chemists Japan 43: 254-259.

12. Inayoshi K, Ishida T, Maeda K, Sugiyama Y, Akiyama $Y$ (2011) Improvement of the clearance pathway prediction by using active learning. IPSJ SIG Technical Reports 7: 35-42.

13. Osawa I and Tomino $Y$ (2012) Excretion of the drug in kidney -excretion from kidney (water solubility). Nihon Jinzo Gakkai Shi 54: 977-980.

14. Suzuki T, Toyohara T, Abe T (2011) Uremic removal which applied transporter function. Annual Review The Kidney 247-256.

15. Sugiyama $Y$ (2010) In Forefront of prediction study on gastrointestinal absorption of drugs: Examples of organizational utilization of PAMPA. Genetic medicine, Medical do Press,Osaka.

16. Ito S, Ando H, Ose A, Kitamura Y, Ando T, et al. (2013) Relationship between the urinary excretion mechanisms of drugs and their physicochemical properties. Journal of Pharmaceutical Sciences 102: 3294-3301.

17. Kito T and Kusuwara H (2013) Kidney's drug transporter and drug-induced renal nephrotoxicity. The Pharmaceuticals 55: 2355-2362.

18. Umetsu R, Nishibata Y, Abe J, Suzuki Y, Hara H, et al. (2014) Evaluation of the association between the use of oral anti-hyperglycemic agents and hypoglycemia in Japan by data mining of the Japanese Adverse Drug Event Report (JADER) database. Yakugaku Zasshi 134: 299-304 